\section{A thirteen year old female with primary T-cell rich B-cell lymphoma of bone masquerading as chronic recurrent multifocal osteomyelitis}

Saadiya A. Haque, ${ }^{1}$ Aziza Shad, ${ }^{3}$ Metin Ozdemirli, ${ }^{1}$ Victoria K. Shanmugam, ${ }^{2}$ Bhaskar Kallakury'

'Department of Pathology; 'Division of Rheumatology; ${ }^{3}$ Department of Pediatric Oncology, Georgetown University Hospital, Washington, DC, USA

\section{Abstract}

Primary lymphoma of the bone (PLB) accounts for $2 \%$ of all non-Hodgkin's lymphomas, and until recently it had not been well characterized in literature. Most cases present in adulthood (average age 50), with localized painful lesions in the long bones, cranium, or axial skeleton.

We describe a case of multifocal PLB in an adolescent female. In this case, the initial presentation, with migratory large joint polyarthralgias and bone pain, mimicked chronic recurrent multifocal osteomyelitis (CRMO). Had a biopsy not been performed the diagnosis would have been missed.

\section{Case Report}

A 13-year old African-American female, with no significant past medical history, presented with migratory large joint polyarthralgias and bone pain. Her symptoms were initially localized to the right ankle and retrocalcaneal region, with constant pain which severely limited her mobility. There was no swelling or erythema, but the patient did comment that the affected areas felt warm. Ibuprofen gave some symptomatic relief. After a few months, the ankle pain resolved, and she developed pain and warmth of the left knee. The pain in this region lasted approximately one month before remitting spontaneously. On initial presentation to the Rheumatology Division of our facility, approximately 18 months after the onset of symptoms, she was complaining of severe pain in the right ribs, right knee, and left groin. She denied weight loss, night sweats, or skin lesions.

Physical examination revealed normal vital signs. She had no evidence of lymphadenopathy. Cardiac and pulmonary examinations were within normal limits. Abdominal examination was without organomegaly. Evaluation of the joints revealed no active synovitis, and all joints had a full range of motion.

Laboratory evaluation revealed a microcytic anemia. Her white cell count, chemistry, and liver function tests were within normal limits. Full autoimmune panel including ANA, doublestranded DNA, rheumatoid factor, and anticyclic citrullinated peptide, and ACE level were within normal limits. Both erythrocyte sedimentation rate and $\mathrm{C}$-reactive protein were elevated at $42 \mathrm{~mm} / \mathrm{hr}$ and $105.6 \mathrm{mg} / \mathrm{L}$, respectively. Vitamin D level was slightly low at 21.8 $\mathrm{ng} / \mathrm{mL}$.

A skeletal survey revealed mixed sclerotic and lytic lesions (Figure 1) involving the calvarium, proximal metaphyses of the humeri,

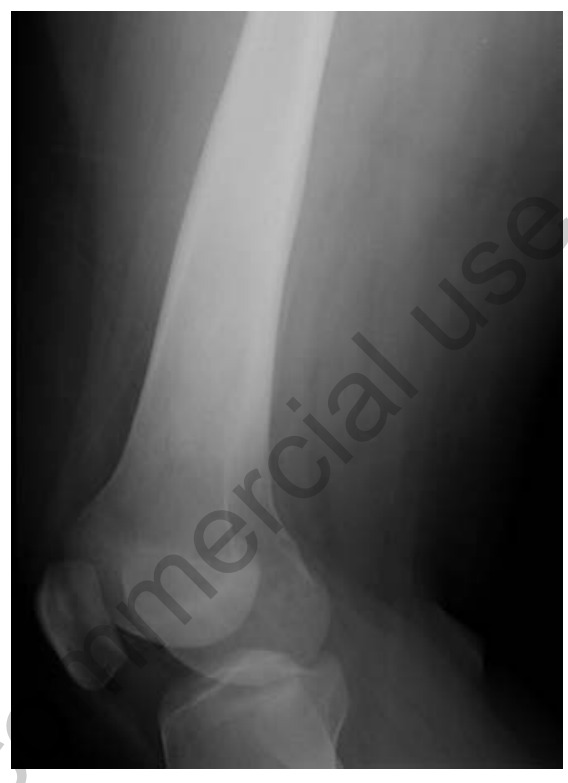

Figure 1. X-Ray: expansile sclerotic deformity of the distal femur.
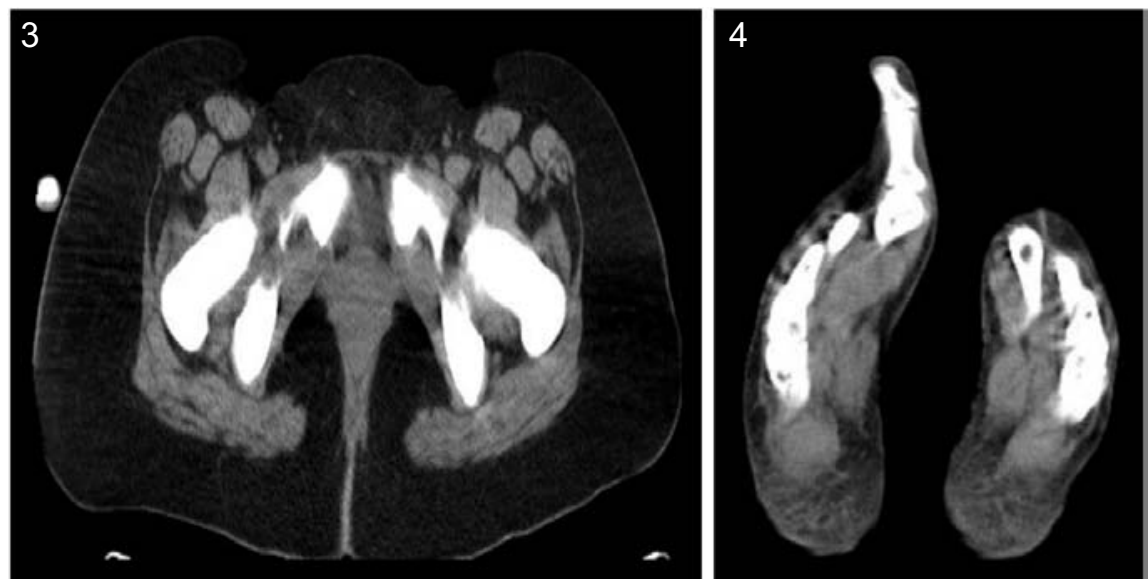

Figure 3 and 4. NM PET scan: innumerable foci of increased radiotracer uptake throughout the skeleton.
Correspondence: Saadiya Haque, Georgetown

University Hospital, Washington DC, USA

E-mail: saadiyahaque@yahoo.com

Key words: chronic recurrent multifocal osteomyelitis, primary lymphoma of bone, bone pain, adolescent.

Received for publication: 5 September 2009.

Revision received: 9 September 2009.

Accepted for publication: 9 September 2009.

This work is licensed under a Creative Commons Attribution 3.0 License (by-nc 3.0).

(C)Copyright S.A. Haque et al., 2009

Pediatric Reports 2009; 1:e3

doi:10.4081/pr.2009.e3

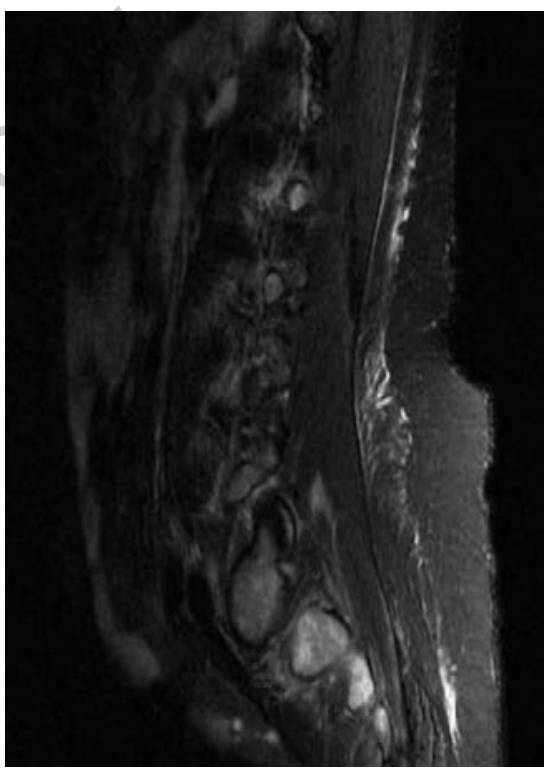

Figure 2. MRI spine: abnormal enhancement of vertebral bodies. 

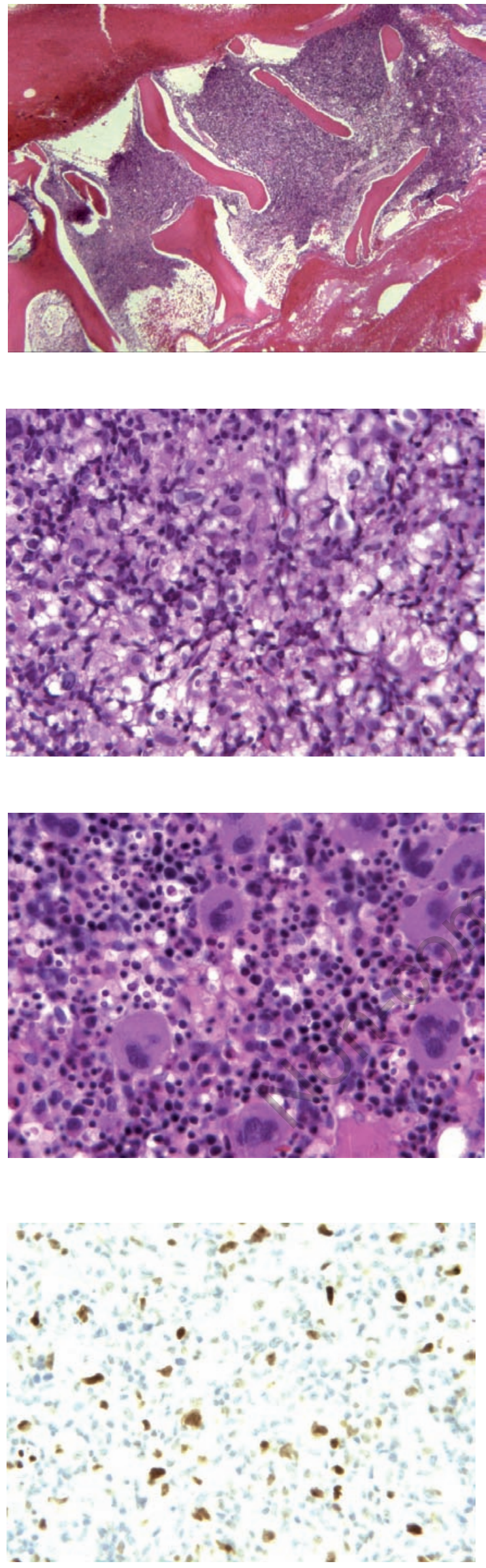

Figure 5. Hypercellular bone marrow.

Figure 6. Atypical lymphoid infiltrate.

Figure $7 . \quad$ Trilineage hematopoiesis.

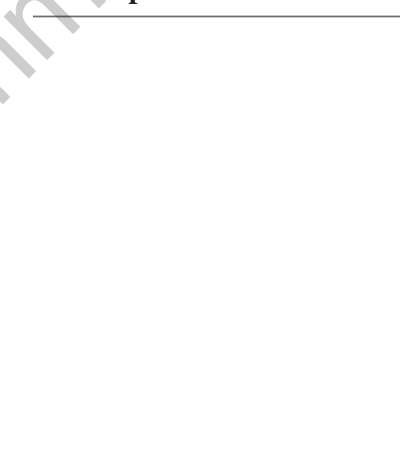

Figure 8. bcl-6. proximal left clavicle, right femoral neck, bilateral distal femurs, bilateral tibias, and right talar dome. The affected bones demonstrated involvement of the epiphysis, metaphysis, and diaphysis with minimal periosteal reaction. Magnetic resonance imaging of the right foot revealed abnormal marrow signal in the tibia, calcaneus, talus, and cuboid (Figure 2). Nuclear bone scan demonstrated abnormal radiotracer uptake in the skull, spine, hips, femurs, tibias, and feet, corresponding to the lesions on the skeletal survey (Figure 3 and 4).

The indolent presentation, with migratory relapsing and remitting bone pain, along with the radiographic findings of sclerotic and lucent lesions, suggested a diagnosis of chronic recurrent multifocal osteomyelitis. Bone marrow biopsy was performed to exclude malignancy, and this identified a primary T-cell rich diffuse large B-cell lymphoma of bone.

H\&E stained sections of the bone marrow biopsy revealed a hypercellular marrow, showing large atypical lymphoid cells with irregular nuclei and small nucleoli surrounded by a background of small mature lymphocytes and eosinophils (Figures 5 and 6). Other areas of the biopsy showed trilineage hematopoiesis (Figure 7). The immunohistochemical analysis revealed the large atypical cells to be positive for CD20, LCA, CD23, and bcl-6 with a significantly high Ki-67 proliferation index (Figure 8). The background hematopoietic cells demonstrated a T-cell rich component. The atypical cells were negative for T-antigens, CD10, CD21, bcl-2, CD34, TdT, CD30, CD15, CD1a, S100, CD56, and ALK-1. These histological and clinical findings are consistent with primary T-cell rich diffuse large B-cell lymphoma (DLBCL) of bone.

\section{Discussion}

Chronic recurrent multifocal osteomyelitis (CRMO) is a rare childhood disease with a female predominance, characterized by bone pain, fever, and a chronic relapsing and remitting course. It is frequently associated with other inflammatory conditions such as psoriasis or pustulosis palmoplantaris and is thought to represent the pediatric presentation of SAPHO syndrome (synovitis, acne, pustulosis, hyperostosis, and osteitis). Typically CRMO presents with insidious pain and tenderness. Fever and swelling are not always present. Lesions usually localize to the metaphyses of long bones and the clavicle, and the most consistent laboratory finding is elevation of the erythrocyte sedimentation rate. Radiographs typically show irregular osteolytic lesions with surrounding sclerosis, and lesions may or may not be active on nuclear scans using Tc-99. ${ }^{1}$

Major and minor diagnostic criteria have 
Table 1. Major and minor diagnostic criteria of CRMO.

\begin{tabular}{ll}
$\begin{array}{l}\text { Major diagnostic criteria } \\
\begin{array}{l}\text { Radiologically proven osteolytic/ } \\
\text { osteosclerotic bone lesions }\end{array}\end{array}$ & $\begin{array}{l}\text { Normal blood cell count and good general state } \\
\text { of health } \\
\text { Multifocal bone lesions }\end{array}$ \\
\hline $\begin{array}{l}\text { CRP and ESR mildly to moderately elevated } \\
\begin{array}{l}\text { Sterile bone biopsy with signs of } \\
\text { inflammation and sclerosis }\end{array}\end{array}$ & $\begin{array}{l}\text { Course longer than } 6 \text { months } \\
\text { Hyperostosis }\end{array}$ \\
\hline & $\begin{array}{l}\text { Association with other autoimmune disease } \\
\text { Grade I or II relatives with autoimmune or } \\
\text { inflammatory disorder or non-bacterial osteitis }\end{array}$ \\
\hline
\end{tabular}

been proposed for $\mathrm{CRMO}^{2}$ and are listed in Table 1. A diagnosis of non-bacterial osteitis can be reached if two major criteria or one major criterion and three minor criteria are present.

In this case, the presence of multifocal bone disease with radiological evidence of osteolytic and sclerotic lesions, along with the elevation of inflammatory markers, and the prolonged clinical course were all suggestive of CRMO. Due to the lack of response to non-steroidal anti-inflammatory agents, empiric treatment with steroids or tumor necrosis blocking agents was considered, and had these agents been commenced prior to biopsy they could have led to further delays in diagnosis. This case underscores the importance of performing biopsy in all cases of suspected CRMO.

Primary lymphoma of the bone is a rare type of lymphoma, comprising $5 \%$ of all primary malignant bone tumors and $2 \%$ of all extranodal non-Hodgkin's lymphomas. ${ }^{3}$ It should be considered in the differential diagnosis of persistent bone pain in all age groups. The diagnosis of PLB requires a bony lesion with unequivocal histological evidence of lymphoma and without evidence of systemic lymphoma within six months of presentation. Most reported cases have been in adults, particularly in the HIV-positive and immunosuppressed post-transplant populations. The peak incidence of PLB is in the fifth decade with a slight preponderance of men over women (male to female ratio, 1.5-2:1).

The pattern of bone involvement in PLB varies, but most cases involve a single focus of bone involvement in the long bones, cranium, or axial skeleton. The spine is the most common site of axial lesions, and the femur is the most frequent site overall. ${ }^{4}$ As was seen in this case, waxing and waning chronic dull achy pain may be the only clinical finding, and due to this indolent presentation, there is often a delay in diagnosis. Most patients do not have abnormalities on physical examination, ${ }^{4}$ and elevation of erythrocyte sedimentation rate or mild anemia may be the only laboratory abnormalities.

In a retrospective study of 23 patients, Wang et al. reported various radiographic lesions such as invasive, osteolytic, sclerotic, and cystiform expansion. ${ }^{5}$ Typically lesions do not demonstrate periosteal reaction. ${ }^{5}$ Magnetic resonance imaging of spine lesions has been reported as having a heterogeneous appearance with abnormal enhancement of the vertebral bodies. Lesions often demonstrate increased radiotracer uptake on Tc99 nuclear bone scan. The definitive diagnosis of PLB is established with a bone marrow biopsy.

The prognosis of PLB is excellent in adults and even better in pediatric patients when compared to other extranodal lymphomas. Good prognostic indicators include young age, histological types including diffuse large B-cell lymphoma and Hodgkin's lymphoma, subtypes including T-cell/histiocyte rich B-cell lymphoma, CD10 positivity, and single foci of bone involvement. The clinical stage, as dictated by the Ann Arbor classification, appears to be the most important prognostic indicator, and the overall 5-year survival from PLB is 54\%. ${ }^{6}$

Diffuse large B-cell lymphoma (DLBCL) is the most common histological type (80\%) of PLB. ${ }^{7}$ About $70 \%$ of DLBCL cases are CD10 positive, which is associated with a better response to chemotherapy. However, in pediatric patients, Zhao et al. found that CD10 negativity does not predict a poor prognosis, unlike in adult PLB-DLBCL. ${ }^{7}$ In a retrospective study of 28 patients with PLB, several other histological types were identified including Blymphoblastic lymphoma, anaplastic large cell lymphoma, natural killer/T-cell lymphoma, and Hodgkin's lymphoma. ${ }^{8}$ Of these, the immunoblastic variant of DLBCL and the natural killer/T-cell lymphoma have the worst prognosis. ${ }^{8}$ In contrast, the T-cell rich subtype of DLBCL, which usually occurs in older patients with multi-organ involvement, conveys a more favorable prognosis.

\section{Conclusion}

This is the first reported case of T-cell rich B-cell lymphoma in a previously healthy pedi- atric patient with primary involvement of the bone. It highlights the importance of confirming clinical diagnoses with histopathological evaluation to prevent delays in diagnosis and therapy. The patient presented in this report has since undergone chemotherapy and at the end-of-therapy staging she was in complete remission. Staging work-up included comprehensive physical examination, bilateral bone marrow biopsy and aspirate, radiological studies including whole body whole body nuclear medicine scan, computed tomography of chest, abdomen, and pelvis, and PET scan. At the end of chemotherapy, histological evaluation of bone marrow revealed trilineage hematopoiesis and no evidence of lymphomatous involvement. Imaging studies demonstrated no evidence of metastatic or residual disease. Based on these findings, the patient was considered to be in remission. So far, she has been in remission for 13 months.

\section{References}

1. El-Shanti HI, Ferguson PJ. Chronic recurrent multifocal osteomyelitis: A concise review and genetic update. Clin Orthop Relat Res 2007;462:11-9

2. Jansson A, Renner ED, Ramser J, et al. Classification of non-bacterial osteitis: retrospective study of clinical, immunological and genetic aspects in 89 patients. Rheumatology (Oxford) 2007;46:154-60.

3. Chin HW, McGuire MH, Block M, et al. Primary lymphoma of bone. Nebr Med J 1990;75:303-6.

4. Franczyk J, Samuels T, Rubenstein J, et al. Skeletal lymphoma. Can Assoc Radiol J 1989;40:75-9.

5. Wang SH, Yu L, Wang QS, Zhao Y, Li F. Clinical Analysis of 23 patients with primary non-Hodgkin's lymphoma originated in bone. Zhongguo Shi Yan Xue Ye Xue Za Zhi 2008;16:200-2.

6. Choi JY, Hahn JS, Suh CO, Yang WI. Primary lymphoma of bone - Survival and prognosis. Korean J Intern Med 2002;17: 191-7.

7. Zhao XF, Young KH, Frank D, et al. Pediatric primary bone lymphoma-diffuse large B-cell lymphoma: morphologic and immunohistochemical characteristics of 10 cases. Am J Clin Pathol 2007; 127:47-54.

8. Maruyama D, Watanabe T, Beppu, et al. Primary bone lymphoma: a new and detailed characterization of 28 patients in a single-institution study. Jpn J Clin Oncol 2007;37:216-23. 